\title{
Analysis of the Spatial Pattern of Strawberry Angular Leaf Spot in California Nursery Production
}

\author{
Christophe Gigot, ${ }^{\dagger}$ William Turechek, and Neil McRoberts
}

First and third authors: Quantitative Biology and Epidemiology lab, Plant Pathology Department, University of California, Davis, CA; and second author: U.S. Department of Agriculture-Agricultural Research Service, U.S. Horticultural Research Laboratory, Fort Pierce, FL. Accepted for publication 11 April 2017.

\begin{abstract}
In California, angular leaf spot (ALS) is a common disease in strawberry nursery production, and a major concern for nurseries wishing to export plants. As the spatial pattern of a disease can offer insight into pathogen source, mode of dissemination, and how current crop management practices affect epidemic development, an understanding of the spatial pattern of ALS would allow nursery growers to make informed decisions regarding disease management. Ninety-seven field assessments of disease incidence were performed at different nursery locations in 2014 and 2015 to quantify ALS spatial pattern under commercial conditions. Both point-pattern and geostatistical statistical procedures were used to analyze the data. The spatial pattern of ALS was

characterized by a high degree of heterogeneity, as indicated by high median values of the beta-binomial distribution's theta parameter (0.643), and the index of dispersion, $D$ (4.218). The binary power law provided a robust description of the data with estimated slope and intercept parameters significantly greater than 1 and 0 , respectively $(P<0.001)$. Spatial analysis by distance indices (SADIE) detected significant nonrandom spatial arrangements for $64 \%$ of the data sets. Analysis of directional disease spread showed a strong spatial association between sampling units along the same planting row. This suggests that recurrent crop operations during the growing season play a significant role in ALS spread and should be taken into account to improve disease control.
\end{abstract}

Angular leaf spot (ALS), caused by the bacterium Xanthomonas fragariae, is a common disease of strawberry. The pathogen and disease were first described in the United States in 1962, and strawberry is its only known host (Kennedy and King 1962; Maas et al. 1995). Although the disease may cause substantial yield losses in open-field fruit-production plantings throughout the United States (Roberts et al. 1997), its greatest economic impact is on the nursery industry where the pathogen's quarantine status affects the export market of plants notably destined for Mexico and the European Union (van der Gaag et al. 2013). The movement of infected plant material is considered the primary method by which the pathogen is introduced in both fruit and nursery production fields (Pooler et al. 1996; Roberts et al. 1998; Wang and Turechek 2016). Water-splash dispersal occurring during rainfall or overhead irrigation events can be responsible for secondary infections. The viscous bacterial exudates that may be visible on leaf undersides under high-moisture conditions provide inoculum in the field (Maas 1998; Maas et al. 1995).

Copper-based products may be applied to strawberry to offer some protection, but their use is typically limited because of phytotoxicity. Available sources of host resistance have been identified, but these have not yet been bred into commercially available cultivars (Bestfleisch et al. 2015). Preplanting heat treatment has proven to be efficient at reducing contamination of nursery stock, but the technique has not been adopted commercially because a practical method for implementation has yet to be developed (Turechek and Peres 2009; Turechek et al. 2013). In practice, ALS management in nursery fields is based on a combination of approaches such as chemical treatment (e.g., copper), limited usage of overhead irrigation, and physical removal of symptomatic plants and debris to reduce inoculum sources.

Despite regular observations of ALS in strawberry fields extending over several decades, the epidemiology of ALS remains poorly characterized. In particular, no extensive quantitative data on its spatial pattern in nursery fields are available. Such information is

${ }^{\dagger}$ Corresponding author: C. Gigot; E-mail address: chgigot@ucdavis.edu

(C) 2017 The American Phytopathological Society often a prerequisite to, for example, designing better disease management programs, developing more accurate sampling plans to assess the true disease intensity, or disentangling the main factors responsible for disease spread. Different families of statistical procedures have been developed in order to retrieve relevant epidemiological information from spatially explicit assessments of plant disease incidence (Madden and Hughes 1995; Madden et al. 2007). Point-pattern techniques characterize small-scale patterns and are based on counts of diseased individuals (e.g., symptomatic plants or leaves) within sampling units (e.g., quadrats) containing a set number of individuals. They provide a direct measurement of heterogeneity and variability of the data at or below the physical size of the sampling unit, and include distributional analyses and use of the binary power law. Geostatistical tools, such as autocorrelation and spatial analysis by distance indices (SADIE) (Li et al. 2012; Perry 1995; Perry et al. 1999), characterize larger-scale patterns. They allow the degree of association of disease intensity between sampling units to be defined, explicitly taking into account their relative spatial positions, and define heterogeneity at the physical scale above the sampling unit, but not larger than the extent of the area sampled. Used in conjunction, point-pattern and geostatistical procedures can provide comprehensive interpretation of disease spatial patterns, allowing determination of, for instance, the degree of aggregation within disease clusters and the degree of anisotropy of higher-order aggregation. They may also give insights into the physical, biological and anthropogenic phenomena responsible for the observed patterns.

The objectives of this study were to (i) quantify the spatial patterns of the incidence of ALS in strawberry nursery fields in California at multiple spatial scales; (ii) determine if these patterns depended on factors such as year and field elevation; and (iii) assess their degrees of anisotropy.

\section{MATERIALS AND METHODS}

Per request of the nurseries involved in this project, neither the names of the nurseries, the names of the strawberry varieties nor the exact locations of the visited fields are disclosed. 
Data collection. Naturally occurring epidemics of ALS were monitored over two distinct growing seasons, from September to December 2014, and from August to December 2015. Different fields managed by two strawberry plant nurseries (named nursery 1 and nursery 2 in this study) in both high-elevation (Northern California) and low-elevation (California's Central Valley) locations were visited to collect disease incidence data. Only "commercial" fields (i.e., the last stage of plant multiplication before sale of the plants for fruit production) that used overhead irrigation were included in the study, because these fields are typical of those where ALS can become a serious problem. In total, 97 field assessments of disease incidence were performed ( 55 in 2014 and 42 in 2015, 43 in high-elevation and 54 in low-elevation fields, and 58 in nursery 1 and 39 in nursery 2).

Sampled fields were composed of beds of strawberry plants. A bed is a group of 10 to 12 rows of mother plants bordered by overhead irrigation lines. The distance between bed centers was typically around $12.5 \mathrm{~m}$. Daughter plants are produced from runners of mother plants and root between the rows, eventually filling the bed surface completely. At each field location, up to 10 evenly spaced rows (one per bed) were selected for observation. Along each row, 20 sampling units spaced about $5 \mathrm{~m}$ apart were sampled and assessed for disease symptoms (i.e., each row was sampled over a distance of about $100 \mathrm{~m}$ ). Special attention was given not to choose rows nearby tractor tracks. As only one row per bed was selected, the actual number of rows assessed for disease incidence in a field was equal to the number of beds (up to 10) in that field (Table 1). A complete data set for a given field corresponded to a lattice of up to 200 sampling units in which adjacent sampling units across beds were about 2.5 times more distant from each other than adjacent sampling units within rows. For each sampling unit, three leaves were arbitrarily collected from the top of the canopy within an approximate $1 \mathrm{~m}^{2}$ quadrat. All nine leaflets (three leaflets per leaf) were assessed for presence or absence of ALS symptoms using the sun to provide a transmitted light source. In total, 137,880 leaflets distributed over 97 data sets (i.e., field assessments) were assessed as either "healthy" or "diseased".

Notation and statistical software. Throughout this study, $n$ is the number of individuals per sampling unit ( $n=9$ leaflets), and $N$, the number of sampling units per data set (i.e., field assessment). In addition, each sampling unit in a data set can be spatially referenced using two indices, $i$ and $j: i$ indicates a row

TABLE 1. Description of the physical layout of the 97 field assessments of strawberry angular leaf spot incidence in different nursery fields in California at both high- and low-elevation field sites in 2014 and 2015

\begin{tabular}{lcc}
\hline Number of beds $^{\mathrm{a}}$ & $\begin{array}{c}\text { Number of } \\
\text { sampling units }^{\mathrm{b}}\end{array}$ & $\begin{array}{c}\text { Percentage of field } \\
\text { assessments }^{\mathrm{c}}\end{array}$ \\
\hline 1 & 20 & $4.1 \%(4 / 97)$ \\
2 & 40 & $9.3 \%(9 / 97)$ \\
3 & 60 & $1.0 \%(1 / 97)$ \\
4 & 80 & $2.1 \%(2 / 97)$ \\
5 & 100 & $5.2 \%(5 / 97)$ \\
6 & 120 & $2.1 \%(2 / 97)$ \\
7 & 140 & $5.2 \%(5 / 97)$ \\
8 & 160 & $11.3 \%(11 / 97)$ \\
9 & 180 & $7.2 \%(7 / 97)$ \\
10 & 200 & $52.6 \%(51 / 97)$ \\
\hline
\end{tabular}

${ }^{a}$ For each field assessment, there were up to 10 adjacent beds selected for observation. A bed is a collection of 10 to 12 adjacent rows of mother plants bordered by overhead irrigation lines. The width of a bed was about $12.5 \mathrm{~m}$. The actual number of beds assessed for disease incidence in a field was equal to the number of beds if there were less than 10 beds.

b Along each row selected for observation (one per bed), 20 evenly spaced sampling units (about $5 \mathrm{~m}$ apart) were sampled for assessment of disease incidence.

${ }^{\mathrm{c}}$ In the notation of this article, each field assessment corresponds to a data set. The actual number of data sets over the total number of data sets is indicated in parentheses. number, and $j$, a sampling unit in row $i . Y_{i, j}$ and $y_{i, j}$ are the number of diseased individuals and the proportion of diseased individuals in the sampling unit at the location $(i, j)$, respectively $\left(y_{i, j}=Y_{i, j} / n\right)$. This means that, for instance, $s_{y}^{2}$ refers to the variance calculated from the proportions of diseased individuals. Except for spatial analysis by distance indices (SADIE), all the statistical analyses were carried out using the statistical software environment $\mathrm{R}$ (version 3.3.3).

Spatial analyses for small-scale patterns. Distributions and aggregation indices. The binomial and beta-binomial distributions (Hughes and Madden 1993; Madden and Hughes 1995) were fitted to the disease incidence data for each individual field assessment. Unlike the binomial, which has a single parameter denoting the probability of disease, the beta-binomial has two parameters: $p$, the expected probability of disease, and $\theta$, a measure of the heterogeneity in disease incidence ( $\theta$ increases with the level of heterogeneity). A good fit to the binomial distribution suggests a random spatial pattern, whereas a good fit to the beta-binomial indicates an aggregated spatial pattern of disease incidence. Beta-binomial parameters were estimated by maximum likelihood using the optim procedure implemented in the $\mathrm{R}$ stats package, as described by Sparks et al. (2008). $\chi^{2}$ goodness-of-fit tests were performed to determine if the binomial or betabinomial distributions fitted the data (Hughes and Madden 1993). Also, a log-likelihood ratio test statistic (LRS) was calculated for each data set to determine if the beta-binomial distribution better explained the observed frequency distribution of diseased leaflets than the binomial distribution. LRS = $2\left(\ln \mathcal{L}_{\text {betabin }}-\ln \mathcal{L}_{\text {bin }}\right)$, where $\mathcal{L}_{\text {betabin }}$ and $\mathcal{L}_{\text {bin }}$ are the likelihood functions for the beta-binomial and binomial, respectively. The LRS has a $\chi^{2}$ distribution with one degree of freedom under the null hypothesis of no difference between the two log-likelihoods (i.e., the beta-binomial distribution does not fit the data better than the binomial).

The index of dispersion for incidence data, $D$, was calculated for each data set as the ratio of the estimated observed variance of $y$ to the estimated variance expected if $y$ was binomially distributed, $s_{y}^{2} / s_{\text {bin }}^{2}$, in which $s_{\text {bin }}^{2}=p(1-p) / n$ and $n=9$ (Madden and Hughes 1995). $D<1$ indicates a regular spatial pattern; $D=1$ indicates a random pattern; and $D>1$ indicates an aggregated pattern. If $N$ is the number of sampling units in the data set, then $D(N-1)$ follows a $\chi^{2}$ distribution with $N-1$ degrees of freedom under the null hypothesis of randomness. $D$ has the interesting characteristic that it is expressible as a function of the beta-binomial parameter $\theta$ (Madden and Hughes 1995) as

$$
D=1+\frac{\theta(n-1)}{\theta+1}
$$

Binary power law. The binary form of Taylor's power law (Taylor 1961) was used to assess the overall degree of heterogeneity in a collection of data sets at the sampling-unit scale. The binary power law describes the relationship between the observed variance of diseased individuals within a data set $\left(s_{y}^{2}\right)$ and the corresponding variance under the assumption that the data have a binomial distribution $\left(s_{\text {bin }}^{2}\right)$ and can be expressed as

$$
s_{y}^{2}=A_{p}\left(s_{b i n}^{2}\right)^{b} \text { with } A_{p}=a n^{b}
$$

where $a$ and $b$ are parameters to be estimated, $s_{b i n}^{2}=p(1-p) / n$ and $n=9$ (Hughes and Madden 1992). If $A_{p}=1$ and $b=1, s_{y}^{2}$ is equal to $s_{b i n}^{2}$, which indicates a random distribution of disease incidence that can be described by the binomial distribution. If $A_{p}>1$ and $b=1$, disease incidence is aggregated and the degree of aggregation is independent of $p$. If $A_{p}>1$ and $b>1$, disease incidence is aggregated and the degree of aggregation changes with $p$ (Madden and Hughes 
1995). The logarithmic transformation of equation 2 gives the following linear model:

$$
\ln \left(s_{y}^{2}\right)=\ln (a)+b \ln [p(1-p)] \text { with } a=A_{p} n^{-b}
$$

in which $\ln (\cdot)$ denotes the natural $\operatorname{logarithm}, \ln (a)$ and $b$ are the intercept and slope of a straight line, respectively. This model can be easily fit to the data by linear regression to estimate the parameters. In our case, each data set was first assigned a weight proportional to its number of sampling units prior to model fitting (Table 1), so that fields sampled more intensively had a greater influence on parameter estimates than fields sampled less intensively.

Additionally, to determine if the power law relationship was affected by year (2014 or 2015), field elevation (high- or lowelevation) and nursery (nursery 1 or nursery 2), categorical variables were constructed for each factor and introduced into the power law model as an intercept term, an interaction with the slope, or both. Significance of the additional term was determined by evaluating the change in deviance. Note that in all cases, the standard errors for $a$ and $A_{p}$ were estimated using the delta-method (Oehlert 1992).

The beta-binomial parameter $\theta$ can be expressed in terms of the binary power law parameters $a$ and $b$ (Madden and Hughes 1995) as follows:

$$
\theta=\frac{a-f(p) / n}{f(p)-a}
$$

where $f(p)=[p(1-p)]^{1-b}$. When $b>1$, equation 4 describes a parabolic-like curve with a maximum value at $p=0.5$, and a horizontal line when $b=1$. The relationship between $\theta$ and $p$ has particular utility for characterizing spatial patterns and designing sampling plans (Madden and Hughes 1995).

Spatial hierarchy analyses. The manner in which the data are collected provides information about aggregation of disease at different levels in a spatial hierarchy (Hughes et al. 1997). For example, a sampling unit (upper level) can be reported as "healthy", if no diseased leaves (lower level) were found within the sampling unit. In this study, the sampling unit is defined by three hierarchical levels: the leaflet level (lowest), the leaf level, and the sampling unit level (highest). Each sampling unit consists of three leaves of three leaflets each. Three hierarchical relationships between disease incidence were considered: leaf and leaflet; sampling unit and leaf; and sampling unit and leaflet. If $h$ is a level in the spatial hierarchy ( 1 being the lower level), then $h=1, h=2$, and $h=3$ indicate the leaflet, leaf and sampling unit levels, respectively. Here $n_{h-1, h}$ is the number of individuals at level $h-1$ contained in a unit at level $h$. In this way, $n_{1,2}=n_{2,3}=3$ (i.e., three leaflets in a leaf, and three leaves in a sampling unit) and $n_{1,3}=n=9$ (i.e., nine leaflets in a sampling unit).

In a pairwise comparison between levels, the probability that an individual at the lower hierarchical level is diseased is denoted $p_{\text {low }}$, and $p_{\text {high }}$ refers to the probability of disease at the higher level. The relationship between these two probabilities can be written as

$$
p_{\text {high }}=1-\left(1-p_{\text {low }}\right)^{\nu}
$$

where $\nu$ is a parameter ranging from 0 to the corresponding number of individuals at the hierarchical level referenced by $p_{\text {low }}$. If the value of $\nu$ is equal to the number of individuals at the lower hierarchical level contained in a unit of the higher level $\left(n_{\text {low }}\right)$, this suggests that there is no aggregation of disease incidence at the lower level. Conversely, a value of $\nu$ less than $n_{\text {low }}$ is indicative of aggregation at that level. The value of $\nu$ can be interpreted as an effective sample size (Hughes and Gottwald 1999; Madden and Hughes 1999) in the statistical sense that its value indicates the number of independent pieces of information at the lower level. Here, the effective sample size concerns the equating of the zero-term of the binomial distribution with the zero-term of an overdispersed distribution, as described in Madden and Hughes (1999). Using the complementary $\log -\log$ transformation, $\operatorname{CLL}(x)=\ln [-\ln (1-x)]$, one can rewrite the Equation 5 as follows (Madden et al. 2007):

$$
\operatorname{CLL}\left(p_{\text {high }}\right)=\ln (\nu)+\operatorname{CLL}\left(p_{\text {low }}\right)
$$

from which the value of $\ln (\nu)$ can be obtained as the intercept of a linear regression when the slope is constrained to 1 . In addition, nested spatial hierarchies (Turechek and McRoberts 2013) can be expressed as

$$
p_{h}= \begin{cases}p_{1} & \text { if } h=1 \\ 1-\left(1-p_{h-1}\right)^{\nu_{h-1, h}} & \text { if } h \geq 2\end{cases}
$$

where $\nu_{h-1, h}$ characterizes the relationship between levels $h-1$ and $h$. It is therefore possible to iteratively connect each adjacent pairs of levels in the hierarchy using

$$
\begin{aligned}
\operatorname{CLL}\left(p_{m}\right) & =\sum_{h=2}^{m} \ln \left(\nu_{h-1, h}\right)+\operatorname{CLL}\left(p_{1}\right) \\
& =\ln \left(\nu_{1, m}\right)+\operatorname{CLL}\left(p_{1}\right), \text { with } \nu_{1, m}=\prod_{h=2}^{m} \nu_{h-1, h}
\end{aligned}
$$

in which $\nu_{1, m}$ represents the effective sample size which characterizes the relationship between two distant levels, namely the lowest $(h=1)$ and the highest $(h=m)$ ones.

Spatial analyses for larger-scale patterns. Spatial analysis by distance indices (SADIE). This geostatistical approach uses the relative locations of the sampling units and the number of diseased individuals (i.e., leaflets) per sampling unit to quantify the spatial arrangement of diseased individuals by calculating the distance to regularity, $D_{a}$ (Perry 1995; Perry et al. 1999). Note that in the context of SADIE analysis, the subscript $a$ stands for "aggregation": there is no connection with the parameter $a$ obtained by regression in the binary power law analysis. Regularity is defined as the state where each sampling unit of a given data set contains the same number of diseased individuals: i.e., the mean number of diseased individuals for this data set. Therefore, complete regularity corresponds to having $p \times n$ diseased individuals in each sampling unit and $s_{y}^{2}=0$. The SADIE procedure uses a transportation algorithm to calculate the distance to regularity $D_{a}$ and uses a randomization test to determine if an observed $D_{a}$ is a particularly small or large value. Randomization tests were based on 200 new arrangements to derive a frequency distribution of $D_{a}$ 's. One-sided tests of spatial randomness were performed from the proportion of randomizations with distance to regularity greater than the $D_{a}$ value obtained from the observed data set.

For each field, the program WSADIE (Li et al. 2012), which implements the SADIE procedure, was used to calculate the original index of aggregation $I_{a}$. This index can be expressed as

$$
I_{a}=D_{a} / E_{a}
$$

where $E_{a}$ is the mean distance to regularity calculated over all the random spatial rearrangements. $I_{a}<1$ suggests a regular spatial pattern; $I_{a}=1$ suggests a random pattern; and $I_{a}>1$ suggests an aggregated pattern.

Directional analysis of disease spread using spatial autocorrelation. Spatial autocorrelation analyses were conducted to determine if the pattern of disease spread was anisotropic. For each field assessment, three spatial autocorrelation statistics were calculated, each one based on a different hypothesis of disease spread: omnidirectional (approximated using a "rook" model), across-beds ("across-bed" model) or within-rows ("within-row" model). The first model leads to isotropic disease patterns, while the latter two are associated with anisotropic patterns.

The distance between two adjacent across-bed sampling units (about $12.5 \mathrm{~m}$ ) was taken as the reference spatial lag. The analysis 
needed to take into account the 2.5-times larger distance between sampling units across adjacent beds compared with adjacent withinrow sampling units. To get a consistent spatial lag between sampling units across-beds and within-rows, virtual sampling units were calculated as follows. For each actual sampling unit, a corresponding virtual sampling unit-located at $12.5 \mathrm{~m}$ from the location of the actual sampling unit on the same row-was given a disease incidence value equal to the weighted-mean of the actual values recorded for the two sampling units nearest to this virtual location (on the same row). The weights of both nearest sampling units in the calculation of the mean were based on their relative distances to the virtual sampling unit.

As autocorrelation analysis strongly relies on the assumption of second-order stationarity (Madden et al. 2007; Reynolds and Madden 1988), the number of diseased leaflets in each sampling unit $\left(Y_{i, j}\right)$ was transformed using the logit function to stabilize the variance in each of the original data sets. The Haldane correction was used to avoid taking the logarithm of zero values and division by zero (Haldane 1956; Reynolds and Madden 1988; Reynolds et al. 1988). Thus, the new values $y_{i, j}^{\prime}$ can be written as $y_{i, j}^{\prime}=$ $\ln \left[y_{i, j}^{\prime \prime} /\left(1-y_{i, j}^{\prime \prime}\right)\right]$, in which $y_{i, j}^{\prime \prime}=\left(Y_{i, j}+0.5\right) /(n+1)$. For a given data set, the spatial autocorrelation coefficient $\left(\hat{r}_{1}\right)$ can be expressed as

$$
\hat{r}_{1}=\left[\sum_{i, j, k, l}^{S_{\text {model }}} \frac{\left(y_{i, j}^{\prime}-p^{\prime}\right)\left(y_{k, l}^{\prime}-p^{\prime}\right)}{N_{\text {model }}}\right] \times\left[s_{y^{\prime}}^{2}\right]^{-1}
$$

where $p^{\prime}$ and $s_{y^{\prime}}^{2}$ denote the average value and the estimated variance of $y^{\prime}$, respectively. For a given spatial autocorrelation model, $N_{\text {model }}$ is the relevant number of pairs of sampling units a specified lag distance apart (i.e., the number of pairs of $y^{\prime}$ values on which the calculation is based for the considered model) (Madden et al. 2007). Just as with $N_{\text {model }}$, the actual set of indices $i, j, k, l\left(S_{\text {model }}\right)$ used to compute $\hat{r}_{1}$ depends on the disease spread hypothesis to be tested (i.e., rook, across-bed, or within-row model).

As with the SADIE procedure, randomization methodology was used to determine if the observed $\hat{r}_{1}$ were particularly small or large. For each data set, 200 random arrangements of the sampling units were generated and three new spatial autocorrelation coefficients (one for each model) were calculated for each of these rearrangements.

Directional analysis of disease spread using spatial prediction of disease intensity. Another geostatistical technique was implemented to provide a comparison for the results obtained with the spatial autocorrelation analysis. While the latter approach focuses on how the covariance between sampling units changes with their spatial separation, the prediction method described here focuses on the values of the sampling units themselves. The basic idea is to determine the extent to which the disease intensity of a sampling unit, $y_{i, j}$, can be predicted by values of disease intensity of nearby sampling units (Sparks et al. 2008).

A predicted disease intensity $\check{y}_{i, j}$ was calculated as the mean of disease intensity of neighboring sampling units. The definition of neighbor depends on the disease spread model to be tested. Given $A_{\text {within }}=y_{i,(j-\text { lag })}+y_{i,(j+\text { lag })}$ and $A_{\text {across }}=y_{(i-\text { lag }), j}+y_{(i+l a g), j}$, the predicted values under the rook, within-row or across-bed model can be expressed as: $\check{y}_{i, j}=\left(A_{\text {within }}+A_{\text {across }}\right) / 4, \quad \check{y}_{i, j}=A_{\text {within }} / 2$, and $\check{y}_{i, j}=A_{\text {across }} / 2$, respectively. A lag of $12.5 \mathrm{~m}$ was used for the same reasons of consistency discussed for spatial autocorrelation computation and so that the two sets of analyses would be comparable.

To test the validity of a disease spread model for a field assessment, the estimated $\breve{y}_{i, j}$ values were compared with the actual $y_{i, j}$ values. A nonrandom spatial pattern is indicated if the estimations of $y_{i, j}$ tended to be nearer the actual values when including disease intensity of neighbors as predictors. If the rook model yields better predictions of disease levels than the other two, this suggests an isotropic disease spread. Conversely, if either the across-bed model or the within-row model best predicts disease intensity values, this indicates that disease predominantly spreads across beds or within rows, respectively. Lin's concordance correlation coefficient $\left(\rho_{c}\right)$, which combines measures of precision and accuracy, was calculated to test the agreement between the actual values $\left(y_{i, j}\right)$ and the predicted values $\left(\check{y}_{i, j}\right)$ by the different models (Lin 1989, 2000; Madden et al. 2007). A perfect concordance between $y_{i, j}$ and $\check{y}_{i, j}$ values would return $\rho_{c}=1$. The value $\rho_{c}$ was calculated using the epi.ccc function of the epiR package (Stevenson et al. 2016).

Note that predictions of the outermost sampling units (i.e., the ones located on the borders of the two-dimensional maps) could not be computed, for at least one of the models, because due to edge effects the complete set of neighboring sampling units was not available. For a given field assessment, the same set of nonedge sampling units was used for all the models, in order to maintain consistent comparisons among them.

As with SADIE and autocorrelation analysis, spatial randomizations were used to determine if observed $\rho_{c}$ were particularly small or large. Two hundred random rearrangements of the sampling units were generated for each data set. New $\rho_{c}$ values, one for each model and spatial arrangement combination, were then calculated using the procedure described earlier.

TABLE 2. Proportions of data sets with significant likelihood ratio test statistic (LRS Sig.), median estimated beta-binomial parameter $(\hat{\theta})$, median index of dispersion for incidence data $(D)$, median index of aggregation of the spatial analysis by distance indices (SADIE) procedure $\left(I_{a}\right)$, and proportions of data sets tested to be significant (Sig., $P<0.05$ ) for each of spatial statistic relative to each disease incidence class of angular leaf spot disease incidence ${ }^{\mathrm{a}}$

\begin{tabular}{|c|c|c|c|c|c|c|c|c|}
\hline \multirow[b]{2}{*}{ Disease incidence class ${ }^{b}$} & \multirow[b]{2}{*}{ Count } & \multirow[b]{2}{*}{ LRS Sig. ${ }^{c}$} & \multicolumn{2}{|c|}{$\hat{\theta}$} & \multicolumn{2}{|c|}{$D$} & \multicolumn{2}{|c|}{$I_{a}$} \\
\hline & & & Median & Sig. & Median & Sig. & Median & Sig. ${ }^{\mathrm{d}}$ \\
\hline$[0.0,0.1]$ & 13 & $12 / 13$ & 0.389 & $11 / 13$ & 3.173 & $12 / 13$ & 1.50 & $7 / 13$ \\
\hline$(0.1,0.2]$ & 18 & $18 / 18$ & 0.736 & $18 / 18$ & 4.330 & $18 / 18$ & 2.01 & $15 / 18$ \\
\hline$(0.2,0.3]$ & 10 & $10 / 10$ & 0.886 & $10 / 10$ & 4.825 & $10 / 10$ & 1.74 & $7 / 10$ \\
\hline$(0.3,0.4]$ & 12 & $12 / 12$ & 0.770 & $12 / 12$ & 4.439 & $12 / 12$ & 1.72 & $8 / 12$ \\
\hline$(0.4,0.5]$ & 15 & $15 / 15$ & 0.642 & $15 / 15$ & 4.154 & $15 / 15$ & 2.01 & $9 / 15$ \\
\hline$(0.5,0.6]$ & 7 & $7 / 7$ & 0.795 & $7 / 7$ & 4.584 & $7 / 7$ & 1.75 & $5 / 7$ \\
\hline$(0.6,0.7]$ & 8 & $8 / 8$ & 0.787 & $8 / 8$ & 4.514 & $8 / 8$ & 2.29 & $5 / 8$ \\
\hline$(0.7,0.8]$ & 6 & $6 / 6$ & 0.647 & $6 / 6$ & 4.220 & $6 / 6$ & 1.22 & $2 / 6$ \\
\hline$(0.8,0.9]$ & 4 & $4 / 4$ & 0.621 & $4 / 4$ & 4.136 & $4 / 4$ & 2.26 & $3 / 4$ \\
\hline$(0.9,1.0]$ & 4 & $4 / 4$ & 0.195 & $2 / 4$ & 2.350 & $4 / 4$ & 1.38 & $1 / 4$ \\
\hline
\end{tabular}

a Data consist of 97 field assessments carried out in 2014 and 2015 in different nursery fields in California.

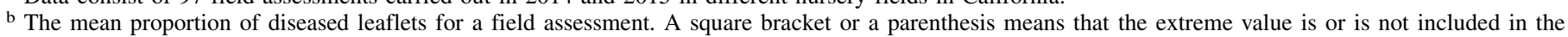
interval, respectively. Summaries are across all data sets.

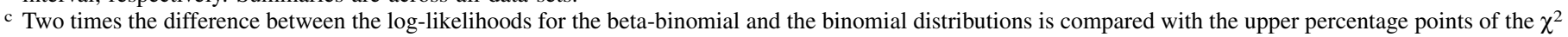
distribution with one degree of freedom $(P<0.05)$.

$\mathrm{d}$ Test for significant spatial aggregation based on the SADIE methodology $(P<0.05)$. 
To ensure that results based on the computation of virtual sampling units were not artificial, we compared the within-row correlation based on the $12.5 \mathrm{~m}$ distance with second- and thirdorder (i.e., 10 and $15 \mathrm{~m}$ lags, respectively) within-row correlation based on the actual observations. Over all the field assessments, the spatial autocorrelation coefficient was $0.386( \pm 0.021)$ and 0.303 $( \pm 0.024)$ for lags of 10 and $15 \mathrm{~m}$, respectively. The autocorrelation coefficient of $0.341( \pm 0.021)$ for the spatial lag of $12.5 \mathrm{~m}$ was not significantly different from the weighted-mean of the coefficients calculated for 10 and $15 \mathrm{~m}$ spatial lags whose estimated value was $0.344( \pm 0.016)(t=0.111, P=0.912)$.

\section{RESULTS}

Spatial analyses for small-scale patterns. Distributions and aggregation indices. Maximum likelihood estimates of the betabinomial parameters $p$ and $\theta$ were successfully calculated for all 97 field assessments (Table 2 ). $\chi^{2}$ goodness-of-fit tests showed that the frequency distribution of diseased leaflets was well $(P<0.05)$ described by the beta-binomial distribution for about $92 \%$ of the data sets; only $2 \%$ of the data sets could be adequately described by the binomial distribution. In addition, the LRS indicated that the frequency distributions of diseased leaflets were significantly better described by the beta-binomial than the binomial distribution in almost all cases. The only LRS which was not significant $(P>0.05)$ corresponded to a data set with a very low mean disease incidence $(\hat{p}=0.022)$. Figure 1 shows a subset of two-dimensional maps of sampling units with corresponding mean incidences $(\hat{p})$, ranging from 0.074 to 0.882 , and degrees of heterogeneity, captured by $\theta$ and ranging from 0.225 to 3.292 . The binomial distribution provided a poor fit to all these examples, whereas the beta-binomial fitted well, especially for both large $\hat{\theta}$ values and bimodal distributions (Fig. 1C to $\mathrm{D}$ and $\mathrm{G}$ to $\mathrm{H}$ ).

Disease incidence and heterogeneity. With a minimum of 0.008 , a maximum of 0.960 , and a median of 0.363 , the observed data sets covered almost the whole range of possible mean disease incidence values for leaflets (Fig. 2A). The lowest recorded $\hat{p}$ value of 0.008 corresponded to a total of 14 diseased leaflets over the 1,800 leaflets assessed for ALS incidence in a field with 200 sampling units.

In comparison with the slightly positively skewed $\hat{p}$ distribution across data sets (Fig. 2A), the frequency distribution of $\hat{\theta}$ was much more positively skewed (Fig. 2B). Two data sets for which $\hat{\theta}=3.292$ (Fig. 1C to D) and $\hat{\theta}=3.332$ that lent to the skewness were associated with a strong aggregation of sampling units with high disease incidence on only one side of the field. This atypical pattern of disease incidence seemed to be related to the specific situation where an adjacent strawberry field exhibited a higher disease intensity and was located upwind. The values of $\hat{\theta}$ ranged from 0.060 to 3.332 , with a median value of 0.643 . Around $96 \%$ of all $\hat{\theta}$ values were significantly greater than $0(P<0.05$ for 93 of the 97 data sets, Table 2). Among the four values of $\hat{\theta}$ that were not significantly different from zero, all had a mean ALS incidence lower than 0.023 or higher than 0.950 . Values of $\hat{\theta}$ were related to $\hat{p}$ in the sense that (i) the lower median $\hat{\theta}$ occurred in the two boundary disease incidence classes (median $\hat{\theta}=0.389$ for 0.0 $\leq \hat{p} \leq 0.1$, and median $\hat{\theta}=0.195$ for $0.9<\hat{p} \leq 1.0$, Table 2 ), and (ii) higher $\hat{\theta}$ were more likely to be found around $\hat{p} \approx 0.5$ (Fig. 3A).

The index of dispersion, $D$, ranged from 1.364 to 7.198 over both years, with a median of 4.218 (Fig. 2C). Only one value of $D$ among the 97 data sets, with a low mean disease incidence $(\hat{p}=0.022)$, was not significantly different from $0(P>0.05$, Table 2$)$. We can note that, even though $D$ and $\hat{\theta}$ values were computed using two different approaches, they match remarkably well over the full range of values as shown by the prediction curve calculated using equation 1 (Figure 3C).

All the point-pattern techniques (i.e., LRS, $\hat{\theta}$, and $D$ ) provided consistent results for more than $96 \%$ of all the data sets (Table 3 ).
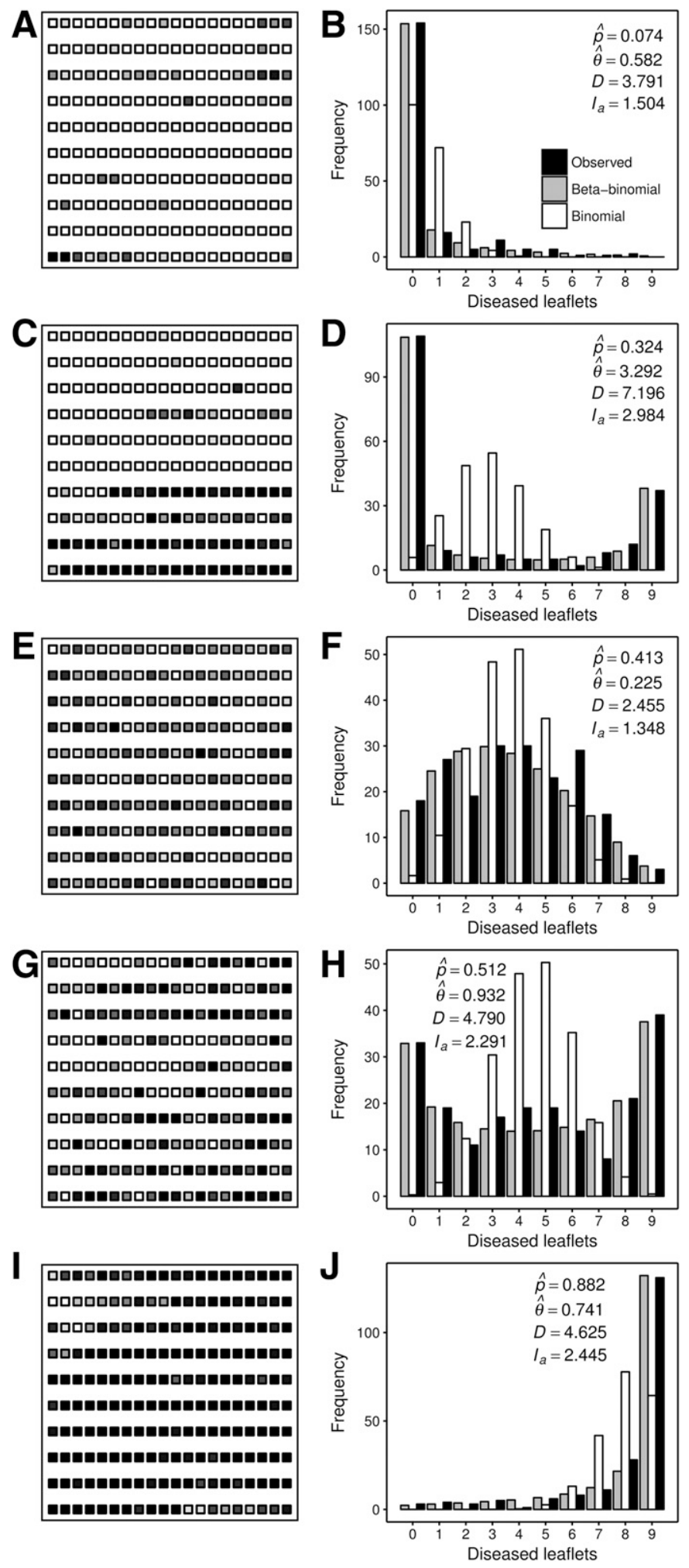

Fig. 1. A, C, E, G, and I, Two-dimensional maps of disease incidence for five example strawberry field assessments with different mean incidences and degrees of aggregation of angular leaf spot. Each small square represents a sampling unit, with $n=9$ leaflets assessed for disease incidence. A darker shade indicates a higher disease incidence. The agronomic rows run horizontally, and the relative distances between sampling units are approximately 5 and $12.5 \mathrm{~m}$ between adjacent within-row and across-bed sampling units, respectively. B, D, F, H, and J, Frequency distributions of the observed number of diseased leaflets per sampling unit (black bars), expected number under the binomial distribution (white bars), and expected number under the beta-binomial distribution (gray bars) for the corresponding maps on the left. The beta-binomial distribution parameters $(\hat{p}$ and $\hat{\theta}$ ), the index of dispersion $(D)$, and the SADIE's index of aggregation $\left(I_{a}\right)$ are specified for each of these examples. 
Binary power law. The binary power law provided a robust description $\left(R^{2}=0.922\right)$ of the relationship between the observed variance of disease incidence for each field assessment $\left(s_{y}^{2}\right)$ and the corresponding theoretical variance under the assumption of random distribution $\left(s_{\text {bin }}^{2}\right)$ on log-log scales (Fig. 4A). Estimates of the two power law parameters ( $a$ and $b$ in equations 2 and 3 ) were $\hat{a}=0.685$ $( \pm 0.051)$ and $\hat{b}=1.213( \pm 0.036)$ (with standard errors in parenthesis). Both $\widehat{A_{p}}=9.846( \pm 1.496)$ and $\hat{b}$ were significantly greater than $1(P<0.001)$. This is an indication that aggregation was dependent on the mean incidence $\hat{p}$ (because $\hat{b}>1$ ). The fact that every field assessment exhibited a certain degree of spatial aggregation can be easily seen in Figure 4A, because every point lies above the binomial (dashed) line which represents the cases where $s_{y}^{2}=s_{\text {bin }}^{2}$.

When considering each year separately, binary power law parameters were estimated to be $\hat{a}=0.672( \pm 0.057)$ and $\hat{b}=1.219$ $( \pm 0.039)$ for 2014 , and $\hat{a}=0.593( \pm 0.102)$ and $\hat{b}=1.097( \pm 0.096)$ for 2015 (Fig. 4B). The estimates of $a$ and $b$ were not significantly different between years $(t=0.680, P=0.50$ for $a$, and $t=1.174, P=$ 0.24 for $b$ ). This was consistent with the absence of a significant year effect in the ANCOVA analysis for the year factor alone $(F=$ 2.691, $P=0.10)$ as well as with its interaction with $\ln \left[s_{\text {bin }}^{2}\right](F=$ $0.473, P=0.49)$. When the data were analyzed by field elevation, the power law parameters were estimated to be $\hat{a}=1.034( \pm 0.155)$ and $\hat{b}=1.381( \pm 0.077)$ for high elevation, and $\hat{a}=0.606( \pm 0.051)$ and $\hat{b}=1.174( \pm 0.040)$ for low elevation (Fig. 4C). The binary power law estimates, $a$ and $b$, were both significantly different between field elevations $(t=2.621, P=0.010$ for $a$, and $t=2.383$, $P=0.02$ for $b$ ). ANCOVA revealed significant differences with the interaction of field elevation factor $\times \ln \left[s_{\text {bin }}^{2}\right](F=10.634, P=$ $0.002)$, but showed no difference for the field elevation factor alone $(F=1.491, P=0.23)$. When considering each nursery separately, the power law parameters were estimated to be $\hat{a}=0.682( \pm 0.093)$ and $\hat{b}=1.250( \pm 0.071)$ for nursery 1 , and $\hat{a}=0.766( \pm 0.054)$ and $\hat{b}=$ $1.225( \pm 0.032)$ for nursery 2 (Fig. 4D). The estimates of $a$ and $b$ were not significantly different between the two nurseries $(t=$ $0.781, P=0.44$ for $a$, and $t=0.318, P=0.75$ for $b$ ). Regarding the ANCOVA analysis, it turned out that the nursery factor alone had a significant effect $(F=11.652, P<0.001)$, but there was no significant effect with its interaction with $\ln \left[s_{\text {bin }}^{2}\right](F=0.107, P=$ 0.74). It should be noticed that no ANCOVA analysis was performed to study the interaction between two or three factors (year, field elevation, and nursery factors) because the observed variances for some situations were too restricted in range, resulting in inaccurate power law parameter estimates (data not shown).

The binary power law parameters were used to compute a prediction curve for the beta-binomial aggregation parameter, $\theta$, based on equation 4 (Fig. 3A). Similarly, the combination of equations 1 and 4 allowed us to compute a prediction curve for the index of dispersion $D$ (Fig. 3E). Both curves in Figure $3 \mathrm{~A}$ and $\mathrm{E}$ exhibited similar quadratic-like shapes, with higher values when $\hat{p} \approx 0.5$ and lower values when $\hat{p} \approx 0$ or $\hat{p} \approx 1$. Even though the predicted $\hat{\theta}$ and $D$ followed the general observed trends with $\hat{p}$, there was considerable variation in the individual $\hat{\theta}$ and $D$ at any given $\hat{p}$, as expected with back-transformation of logarithmic values (Turechek and Madden 1999a, b).

Spatial hierarchy analyses. Effective sample sizes (i.e., $\nu$ values) for the three spatial hierarchy relationships were estimated at $\hat{\nu}_{2,1}=$ $1.672( \pm 0.023)$ for the leaf-leaflet relationship $\left(n_{2,1}=3\right.$ leaflets in a leaf, Figure 5A); $\hat{\nu}_{3,2}=1.825( \pm 0.032)$ for the sampling unitleaf relationship $\left(n_{3,2}=3\right.$ leaves in a sampling unit, Figure $\left.5 \mathrm{~B}\right)$; and $\hat{\nu}_{3,1}=3.064( \pm 0.092)$ for the sampling unit-leaflet relationship $\left(n_{3,1}=n=9\right.$ leaflets in a sampling unit, Figure 5C). Student's $t$ tests showed that all effective sample sizes, $\nu$, were significantly lower
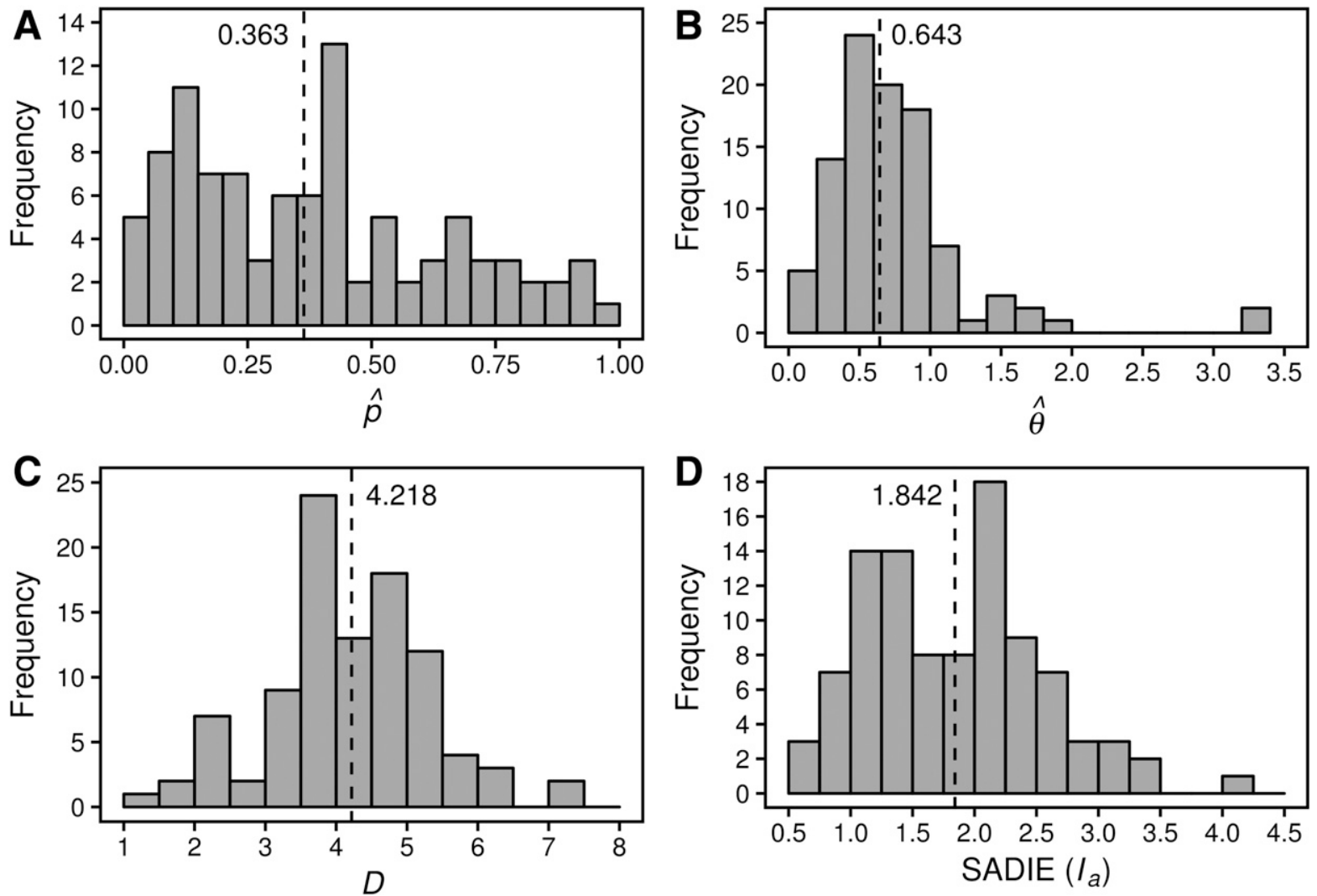

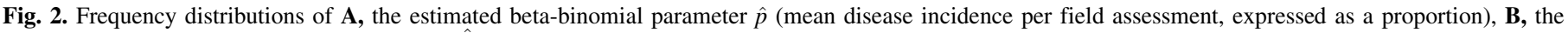

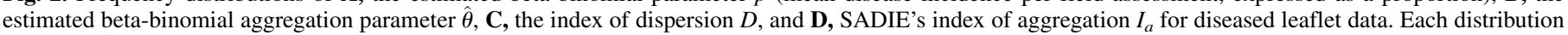

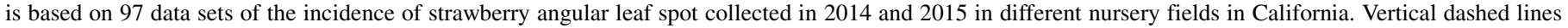
represent the median value of the corresponding statistic. Medians are given numerically on the graphs. 
than the actual corresponding sample sizes, $n(P<0.001)$. Estimated $\hat{\nu}_{2,1}, \hat{\nu}_{3,2}$, and $\hat{\nu}_{3,1}$ were approximately 56,61 , and $34 \%$ of their corresponding $n$, respectively. Using equation 8 with the values of $\hat{\nu}_{2,1}$ and $\hat{\nu}_{3,2}, \hat{\nu}_{3,1}$ was estimated to be $3.052( \pm 0.068)$, which was not significantly different from the actual value of $3.064( \pm 0.092)$ $(t=0.111, P=0.912)$.

Spatial analyses for larger-scale patterns. SADIE. The WSADIE program (Li et al. 2012) was able to compute a distance to regularity
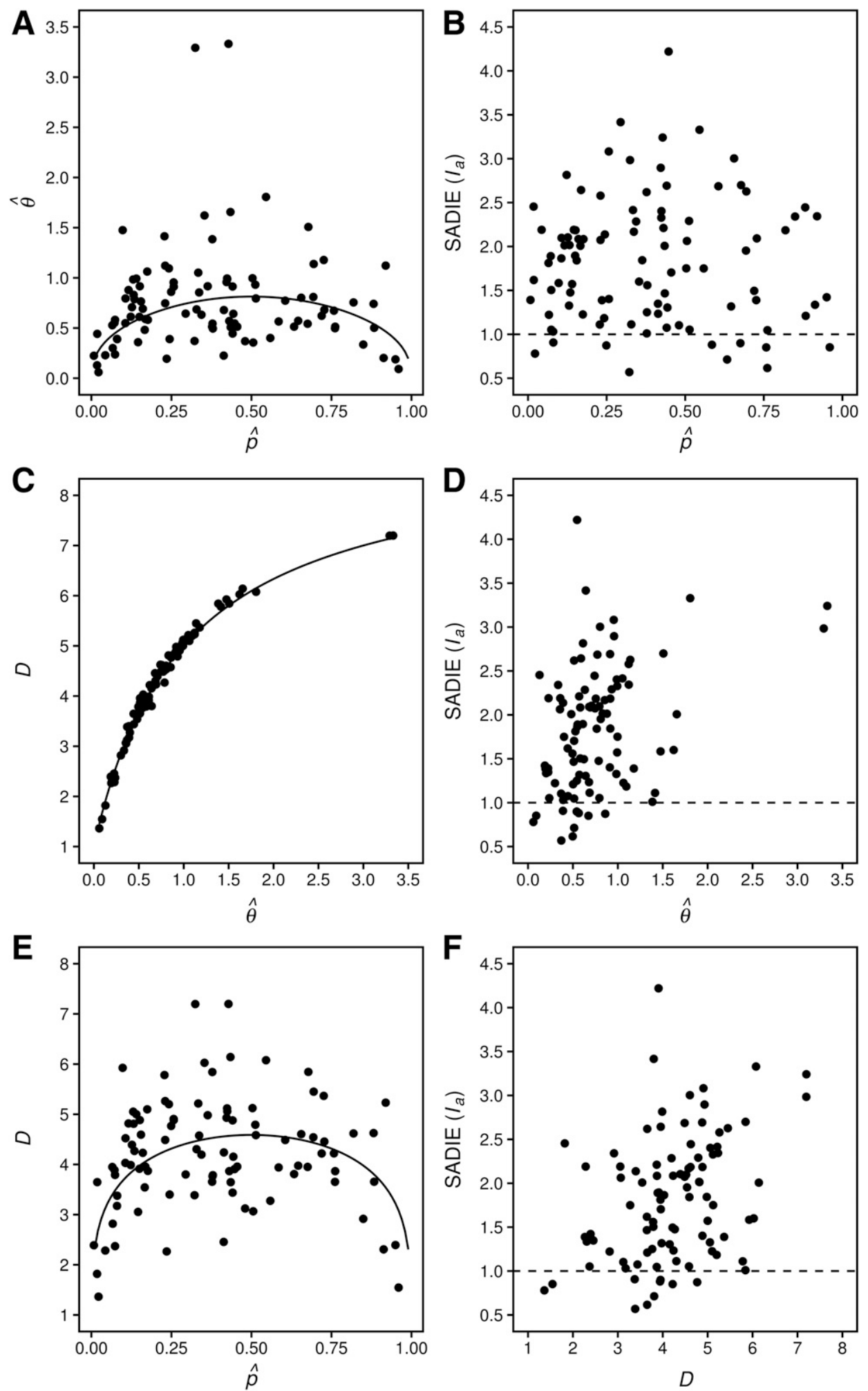

Fig. 3. Relationships between $\mathbf{A}$, the $\hat{p}$ and $\hat{\theta}$ parameters of the beta-binomial distribution, $\mathbf{B}$, the beta-binomial $\hat{p}$ and SADIE's index of aggregation $\left(I_{a}\right)$, $\mathbf{C}$, the beta-binomial $\hat{\theta}$ and index of dispersion $(D), \mathbf{D}$, the beta-binomial $\hat{\theta}$ and SADIE's index of aggregation $\left(I_{a}\right), \mathbf{E}$, the beta-binomial $\hat{p}$ and index of dispersion $(D)$, and $\mathbf{F}$, the index of dispersion $(D)$ and SADIE's index of aggregation $\left(I_{a}\right)$. Each point corresponds to a single field assessment (i.e., a data set) out of the 97 data sets of incidence of strawberry angular leaf spot that were collected in 2014 and 2015 in different nursery fields in California. For graphs with SADIE's index of aggregation $\left(I_{a}\right)(\mathrm{B}, \mathrm{D}$, and $\mathrm{F})$, the dashed lines correspond to the value $I_{a}=1$, which suggests a random pattern. The solid curve in A is derived from equation 4 , which is used to calculate the theoretical value of $\theta$ as a function of $p$ given the binary power law parameters $a$ and $b$. The solid curve in $C$ is derived from equation 1 . The solid curve in $\mathrm{E}$ is derived from the combined use of the equations 4 and 1 to assess $D$ as a function of $p$. 
$\left(D_{a}\right)$ for every data set. $I_{a}$ values (equation 9) ranged from 0.569 to 4.220 , with a median of 1.842 (Fig. 2D). Sixty-two of the 97 computed $I_{a}$ values were significantly different from zero (cf. significant $P_{a}$ values in Table 2). Although there was no a priori reason to observe straightforward relationships between $I_{a}$ and $\hat{p}$, there was a general trend of $I_{a}$ to increase with $\hat{p}$ until $\hat{p} \approx 0.5$, and then to decrease (Fig. 3B; Table 2), sharing this typical behavior with $\hat{\theta}$ (Fig. 3A) and $D$ (Fig. 3E). In addition, $I_{a}$ tended to increase with $\hat{\theta}$ (Fig. 3D) as well as with $D$ values (Fig. 3F), although the relationships were quite variable. SADIE results were in agreement with the point-pattern approaches (i.e., LRS, $\hat{\theta}$, and $D$ ) for more than $60 \%$ of all the field assessments (Table 3 ), which seems to mean that when aggregation occurred, it tended to occur at multiple spatial scales.

Directional analysis of disease spread using spatial autocorrelation. Values of the autocorrelation coefficient $\hat{r}_{1}$, computed based on equation 10 , ranged from -0.491 to 0.525 (with a median

TABLE 3. Percent agreements in the test results for the pairs of spatial statistics used to characterize the spatial aggregation of strawberry angular leaf spot ${ }^{\mathrm{a}}$

\begin{tabular}{lccc}
\hline & $\hat{\theta}$ & $D$ & $I_{a}$ \\
\hline LRS & $96.9 \%(94 / 97)$ & $100.0 \%(97 / 97)$ & $64.9 \%(63 / 97)$ \\
$\hat{\theta}$ & - & $96.9 \%(94 / 97)$ & $68.0 \%(66 / 97)$ \\
$D$ & - & - & $64.9 \%(63 / 97)$ \\
\hline
\end{tabular}

a The actual number of data sets in agreement over the total number of data sets is indicated in parentheses. Data consist of 97 field assessments carried out in 2014 and 2015 in different nursery fields in California. of 0.026 ) for the across-bed model, from -0.117 to 0.825 (with a median of 0.373 ) for the within-row model, and from -0.033 to 0.682 (with a median of 0.200) for the rook model (Fig. 6A; Table 4). Based on estimated $95 \%$ confidence intervals, 29.0, 38.7, and $44.1 \%$ of all the data sets showed that the $\hat{r}_{1}$ 's were significantly different from zero for across-bed, within-row, and rook models, respectively (Table 4).

Directional analysis of disease spread using spatial prediction of disease intensity. The $\rho_{c}$ (concordance correlation coefficient) values between predicted and actual disease incidence data for all the field assessments ranged from -0.169 to 0.304 (with a median of -0.007) for the across-bed model, from -0.093 to 0.616 (with a median of 0.129 ) for within-row model, and from -0.138 to 0.395 (with a median of 0.067), for the rook model (Fig. 6B; Table 4). Estimating 95\% confidence intervals of $\rho_{c}$ based on a resampling procedure, we found that $19.0,25.0$, and $25.0 \%$ of all the field assessments exhibited significant positive $\rho_{c}$ for acrossbed, within-row, and rook models, respectively (Table 4 ).

The percent agreement between directional disease spread analyses based on two different methods (spatial autocorrelation and spatial prediction of disease intensity) was 69.0, 69.0, and $65.5 \%$ for the across-bed, within-row, and rook models, respectively (Table 4). Figure 6C shows $\hat{r}_{1}$ plotted against corresponding $\rho_{c}$ values for all the field assessments where both indices were calculated. The farther from the origin $\left(\hat{r}_{1}=0\right.$ and $\left.\rho_{c}=0\right)$ in the upper-right corner (positive $\hat{r}_{1}$ and $\rho_{c}$ ), the better recorded spatial patterns can be described by the indicated model. Assuming a multivariate normal distribution, the $95 \%$ confidence ellipses for the three disease spread models suggested that the most appropriate model to
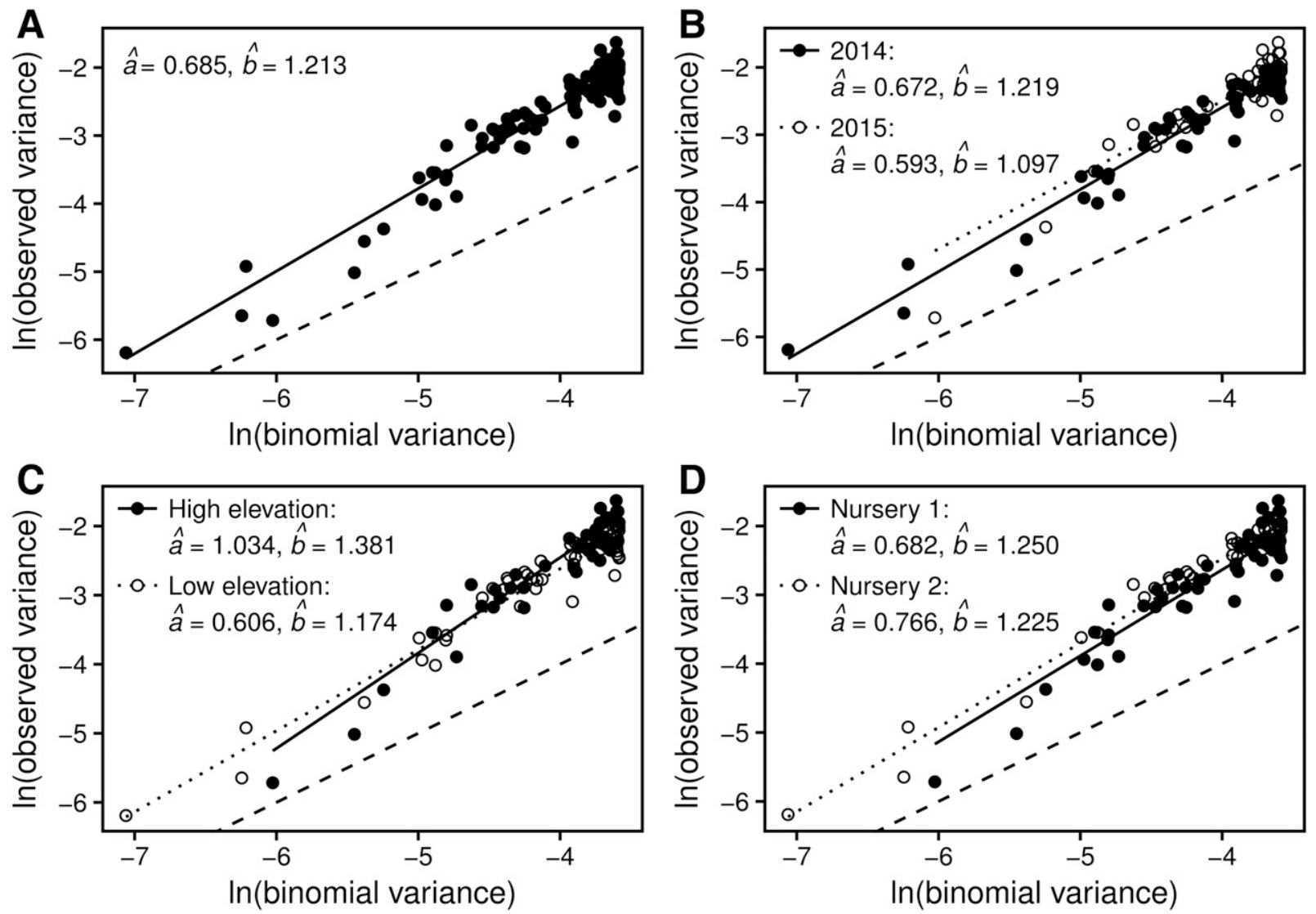

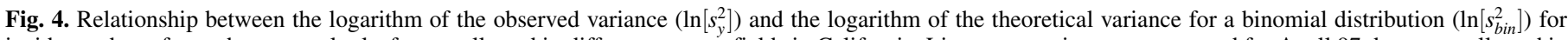

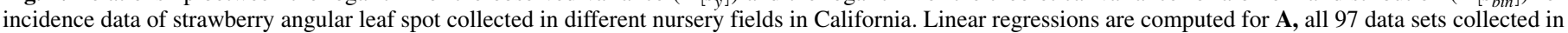

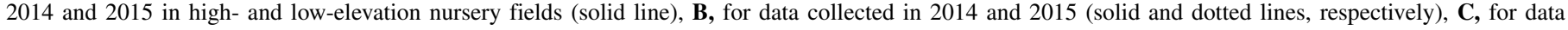

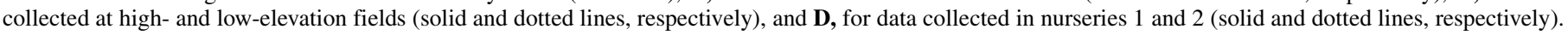

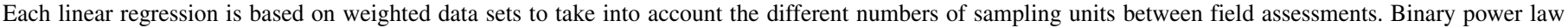

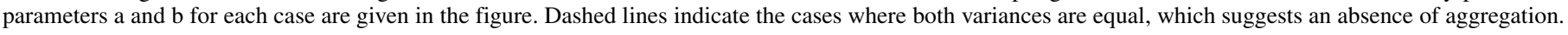


describe ALS incidence patterns in strawberry nursery fields was the within-row model.

\section{DISCUSSION}

The spatial pattern at several scales of strawberry angular leaf spot in California nursery production was characterized from large data sets of field-level observations collected over two growing seasons at several nursery locations. A variety of complementary statistical procedures was used to quantify the heterogeneity and spatial arrangement of disease incidence. Ancillary information, including field location, cropping season and nursery, were analyzed as factors to determine their potential effects on pattern. As spatial arrangements of organisms depend on different scalespecific physical, chemical, biological and anthropic processes (Horne and Schneider 1995), it was possible to not only bring new insights to the understanding of the epidemiology of ALS, but also relevant information that can contribute to the design of improved control strategies.

Unlike most studies of spatial pattern of plant diseases, we observed epidemics that spanned nearly the full range of possible disease incidence values. In particular, observing high values of $\hat{p}$ (near 1.0) is uncommon in the literature. From an analytical perspective, this permitted us to examine or confirm some theoretical aspects of spatial pattern analysis. For example, the relationship between $D$ and $\hat{\theta}$ was visually in good agreement with the theoretical curve over the full range of values (Fig. 3C). This relationship can be described by a straight line for low values as it was noticed for strawberry leaf blight for instance (Turechek and Madden 1999b).
Otherwise, similarly to $D$ and $\hat{\theta}$, a general quadratic-like trend of $I_{a}$ as a function of $\hat{p}$ was seen even though variation of $I_{a}$ at any given $\hat{p}$ was high (Fig. 3B). Such a relationship was not necessarily expected or predicted a priori, because it involves relationships between metrics for different physical scales of pattern. There need not be a relationship between the geo-statistical metric and the point-pattern metric, and future studies with other pathosystems will show if it is general.

The point-pattern analyses indicated that not only did most of the field assessments have significant small-scale aggregation, but that the degree of aggregation was high in comparison with other documented plant diseases (e.g., Madden et al. 1995a, b; Turechek and Madden 1999a, b, 2004). For example, for two other foliar diseases of strawberry studied in fruit production fields (not nursery fields), it was found that median $\hat{\theta}$ was only around 0.10 for leaf blight caused by Phomopsis obscurans (Turechek and Madden 1999b) and 0.20 for fungal leaf spot caused by Mycosphaerella fragariae (Turechek and Madden 1999a). In addition, SADIE showed a high degree of larger-scale aggregation of ALS: in comparison with the median $I_{a}$ of 1.842 calculated for ALS, the median of this index was around 1.13 for leaf blight and 1.38 for leaf spot (Turechek and Madden 1999a, b). Interestingly, the agreement among smallscale and larger-scale approaches (Table 3 ) provided strong evidence of substantial aggregation of ALS under nursery field conditions at multiple spatial scales.

The binary power law indicated that aggregation of disease incidence changed with estimated mean incidence, $\hat{p}$. To place the results from this study in context, the $a$ and $b$ parameters estimated for different plant diseases, compiled by Turechek et al. (2011) from the literature, were plotted in Figure 7. When the estimates of $a$ and
A
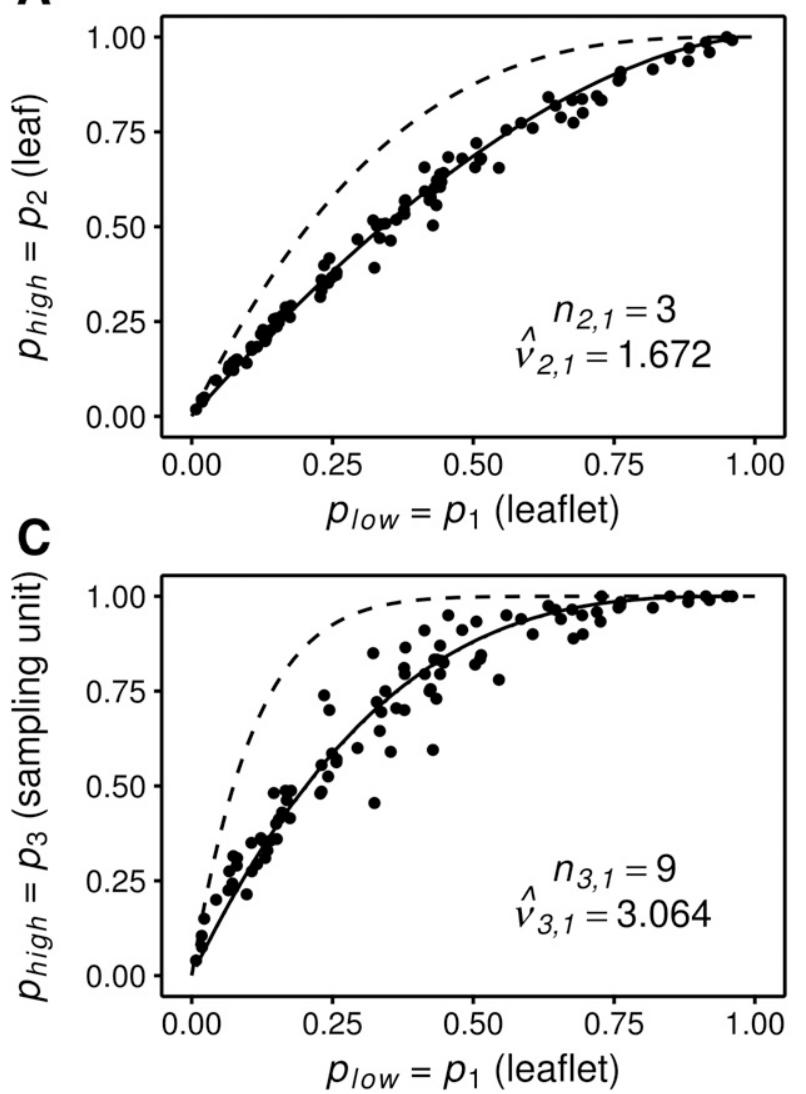

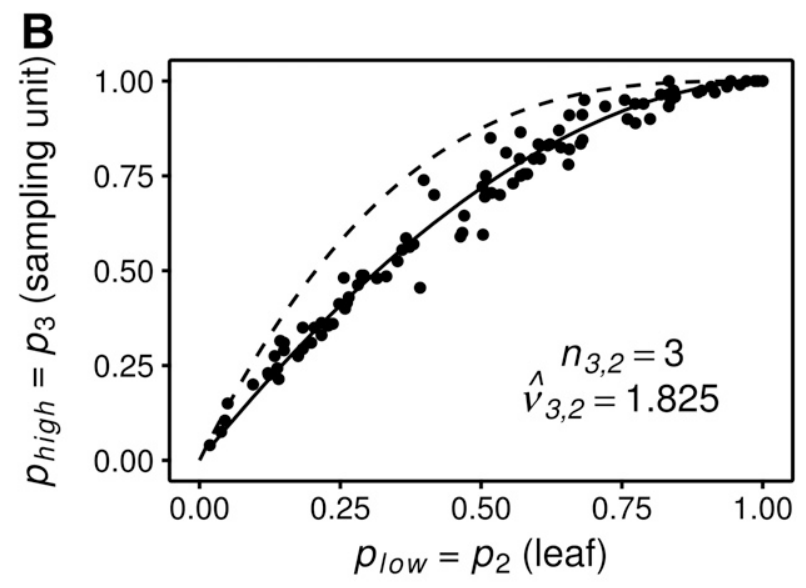

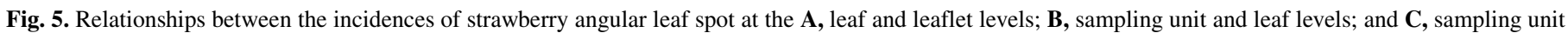

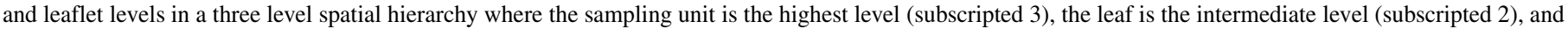

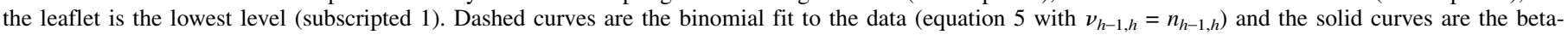

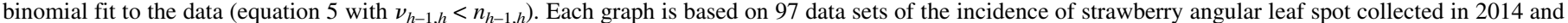
2015 in different nursery fields in California. 
$b$ for ALS were added to this figure, they showed a qualitative agreement with what has been observed for plant pathogens with steep dispersal gradients, typically spread during rainfall or overhead irrigation events. The power law parameters were not significantly different between the years. However, differences in both $a$ and $b$ were found between high- and low-elevation fields, with a higher degree of aggregation found in high-elevation fields. Different factors, such as nursery management practices, susceptibilities of strawberry varieties and environmental conditions (e.g., windy conditions promoting dispersal over longer distances) could explain some of these differences. However, it was not possible to accurately identify which factors were the most important given that our data sets came from actual commercial nursery fields for which we did not choose the input parameters (e.g., management practices and varieties).

The disease incidence data were collected to allow the characterization of ALS incidence in a three-level spatial hierarchy. As in this study, disease incidence data were collected for strawberry leaf blight at the leaflet, leaf and sampling unit levels (Turechek and Madden 1999b, 2003; Turechek et al. 2001). It is possible to make
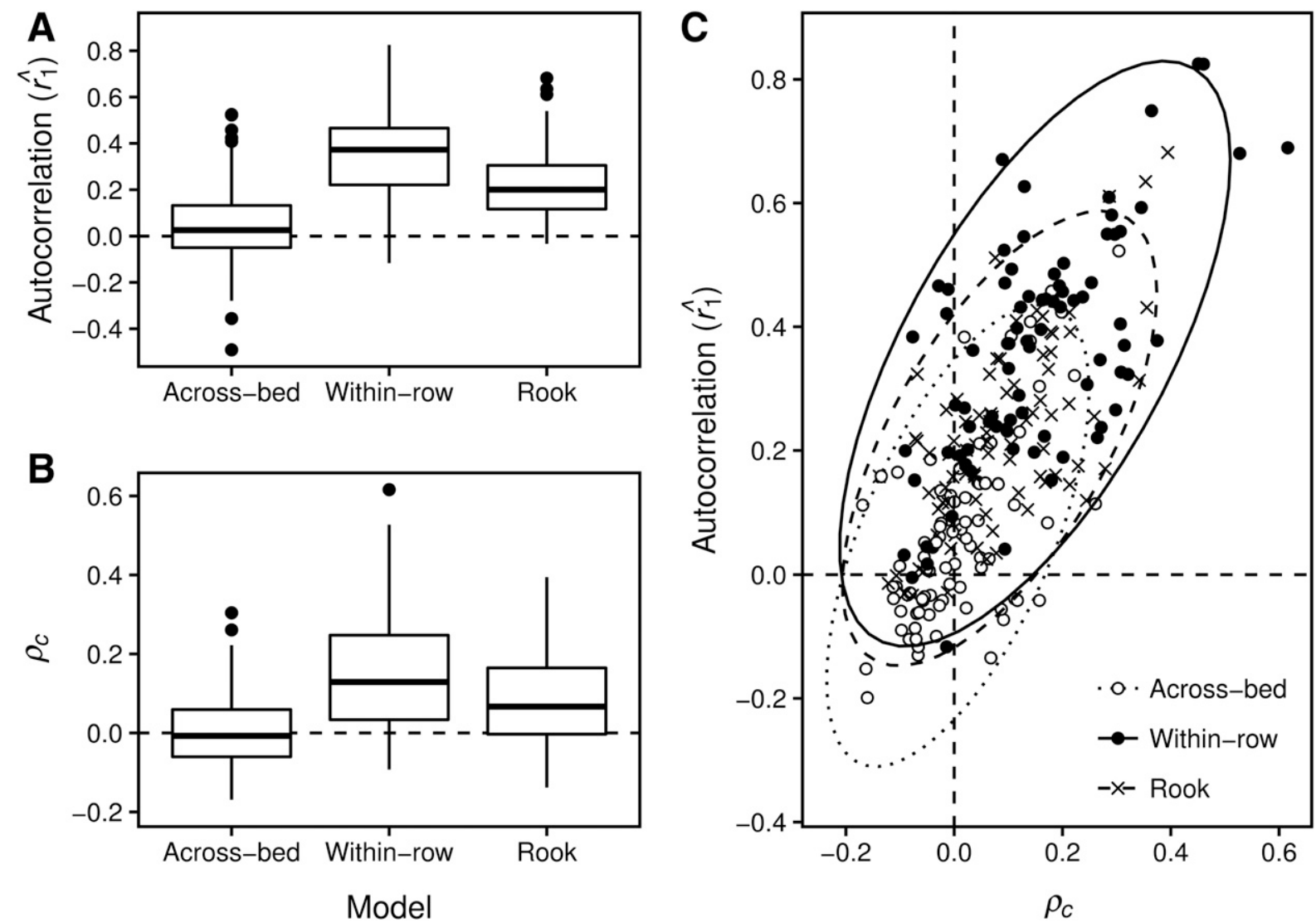

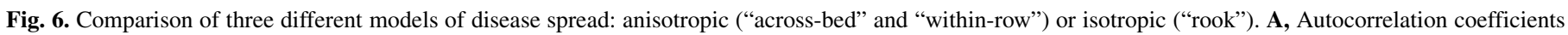

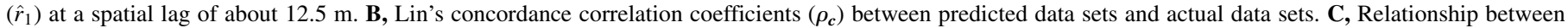

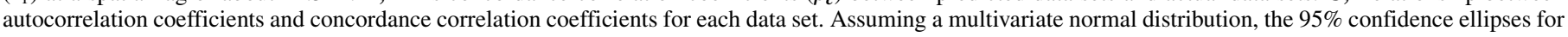

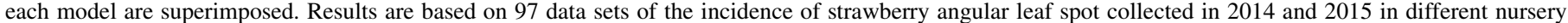

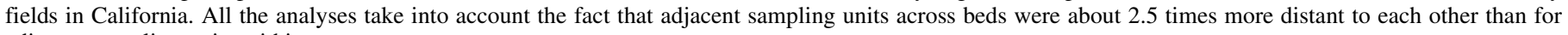
adjacent sampling units within rows.

TABLE 4. Median and significance (Sig., $P<0.05$ ) of statistics relative to spatial association for directional disease spread analyses ${ }^{\mathrm{a}}$

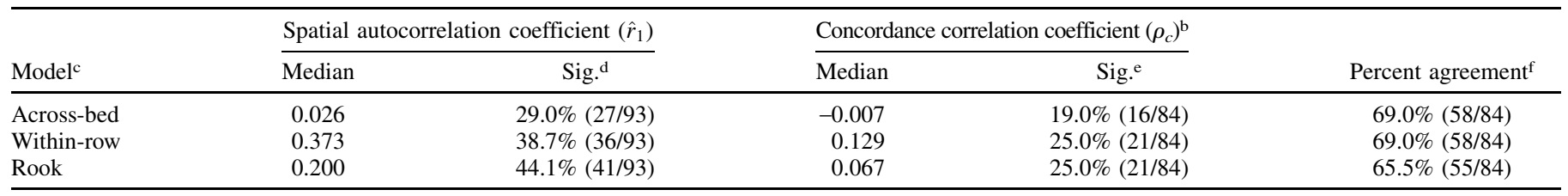

a Results are based on up to 93 field assessments of the incidence of strawberry angular leaf spot collected in 2014 and 2015 in different nursery fields in California.

${ }^{\mathrm{b}}$ Concordance correlation coefficient $\left(\rho_{c}\right)$ was calculated to test the agreement between actual and predicted values of disease intensity using different disease spread models.

${ }^{c}$ Each disease spread model is based on a different hypothesis of predominant directional disease spread: anisotropic across strawberry beds (across-bed model), anisotropic along agronomic rows (within-row model), and isotropic (rook model).

${ }^{d}$ Ninety-three data sets (instead of 97) were used to perform spatial autocorrelation analysis because four of the data sets contained only one row (Table 1), which is insufficient for this analysis.

e Eighty-four data sets (instead of 97) were used to perform spatial prediction of disease intensity because 13 of the data sets contained less than three rows (Table 1 ), which is insufficient for this analysis.

${ }^{f}$ The 84 data sets used in both directional disease spread procedures were used to compute percent agreement for the different disease spread models. The actual number of data sets in agreement over the total number of data sets is indicated in parentheses. 
comparisons between the spatial hierarchy relationships of these two strawberry diseases using the concept of relative effective sample size. Using values extracted from the fitted curves (derived from effective sample size estimation) in Figure 1 of Turechek and Madden (2003) and based on equation 6, we estimated the following effective sample sizes: $\hat{\nu}_{3,2}=4.13$ for the sampling unit-leaf relationship $\left(n_{3,2}=5\right.$ leaves in a sampling unit), and $\hat{\nu}_{3,1}=7.70$ for the sampling unit-leaflet relationship $\left(n_{3,1}=15\right.$ leaflets in a sampling unit). Using equation 8 , the missing effective sample size, for the leaf-leaflet relationship, was estimated to be $\hat{\nu}_{2,1}=\hat{\nu}_{3,1} / \hat{\nu}_{3,2}=$ $1.86\left(n_{2,1}=3\right.$ leaflets in a leaf $)$. With these values, the relative effective sample sizes for strawberry leaf blight were found to be as follows: $\hat{\nu}_{2,1} / n_{2,1}=0.62$ for the leaf-leaflet relationship, $\hat{\nu}_{3,2} / n_{3,2}=$ 0.83 for the sampling unit-leaf relationship, and $\hat{\nu}_{3,1} / n_{3,1}=0.51$ for the sampling unit-leaflet relationship. The corresponding values for ALS were 0.56, 0.61, and 0.34, respectively. Irrespective of the levels of the spatial hierarchy, the relative effective sample sizes were always higher for leaf blight than for ALS (i.e., 1.11, 1.36, and 1.50 times higher for the leaf-leaflet, sampling unit-leaf, and sampling unit-leaflet relationships, respectively). This means that the number of independent pieces of information at the lower level was systematically lower for ALS than for leaf blight, which can be explained by a higher degree of small-scale aggregation at the lower level in the hierarchy. Compared with other documented pathosystems, such as Citrus tristeza virus disease (Hughes and Gottwald 1999) and Plum pox virus disease (Hughes et al. 2002) for which the relative effective sample size ranged between 0.8 and 0.9 , strawberry ALS (and strawberry leaf blight to a lesser extent) exhibited a higher degree of aggregation at the lower levels. This may be at least partly due to the physical size of the individual hosts which plays a role in pathogen dispersal processes (Garrett and Mundt 1999), and actual dispersal processes involved in field nursery production systems. Thus, spatial hierarchy analyses showed that ALS incidence under nursery field conditions was characterized by a very high degree of information redundancy for the three studied hierarchy relationships.

The autocorrelation coefficient $\left(\hat{r}_{1}\right)$ for the within-row model was quite large compared with other strawberry diseases for which incidence data were collected along rows. For strawberry leaf blight, $\hat{r}_{1}$ ranged from -0.27 to 0.52 , with a median of 0.00 (Turechek and Madden 1999b), and for leaf spot, $\hat{r}_{1}$ ranged from -0.17 to 0.85 , with a median of 0.15 (Turechek and Madden 1999a). Although we worked with a five times greater distance between adjacent sampling units (i.e., $12.5 \mathrm{~m}$ instead of $2.5 \mathrm{~m}$ as in the two mentioned studies), the median $\hat{r}_{1}$ of 0.373 for ALS was higher than for published leaf blight and leaf spot data. Thus, there was strong evidence that ALS was characterized by a strong correlation between disease status of leaflets along the rows. In addition, autocorrelation analyses showed that there were significant differences between the within-row and across-bed models for ALS. This contrasts with Turechek and Madden (1999b) who found little directional influence for strawberry leaf blight. Thus, in contrast with strawberry leaf blight in fruit production fields for which data collected along a one-dimensional transect were sufficient to fully characterize its isotropic spatial pattern, a two-dimensional sampling process was needed to capture main features of the anisotropic spatial pattern of ALS under nursery field conditions. Spatial prediction of disease intensity (based on different definitions of neighboring sampling units to be used to predict disease intensity) confirmed autocorrelation results regarding two-dimensional

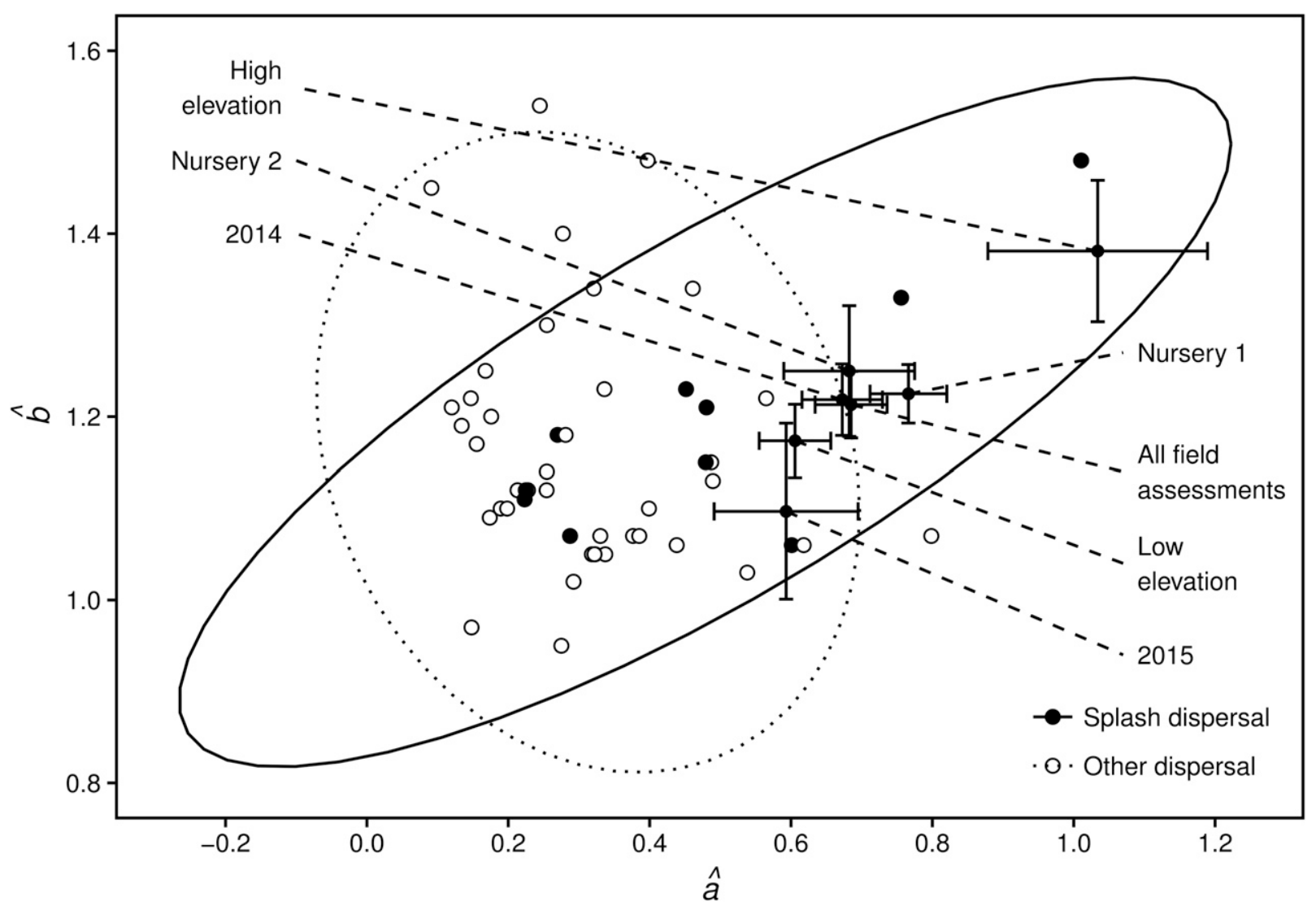

Fig. 7. Comparison between the estimated parameters $a$ and $b$ of the binary power law for different pathosystems extracted from Table 1 in Turechek et al. (2011). Solid symbols indicate estimates from pathosystems whose prevailing mode of dispersal is by splashing water; open symbols indicate all other modes of dispersal. Assuming a multivariate normal distribution, the $95 \%$ confidence ellipses are superimposed. The parameters estimated in this study for angular leaf spot correspond to the points with error bars (standard errors) and are based on analysis of 97 data sets collected in 2014 and 2015 in different nursery fields in California. Estimated parameters considering all the field assessments together, distinguishing years, field elevations, or nurseries, are represented. 
spatial pattern of diseased leaflets. It should, however, be noted that the concordance correlation coefficients $\left(\rho_{c}\right.$, ranging from -0.169 to 0.616 ) were low most of the time, indicating a large component of variation around the linear trends identified by the models.

Both directional disease spread analyses showed that the withinrow model could explain a significantly larger part of the variation in the data than the across-bed and rook models. Conversely, with many $\hat{r}_{1}$ and $\rho_{c}$ values around 0 , the across-bed model was the least able to explain the observed two-dimensional patterns (Fig. 6). Considering the autocorrelation and linear prediction results together, it can be concluded that the data collected over two years, in different nursery fields, showed that ALS spatial pattern exhibited strong anisotropy, with a tendency for the disease to spread primarily along rows rather than across beds. The observed anisotropy in the spatial spread may be due to the combination of several factors over the growing season. First, plants with ALS symptoms may initially appear at apparently random locations in fields owing to the not well-characterized initial occurrences of infected plants. But also, a batch of already infected (but asymptomatic) plants could sometimes be planted in a row (or in a couple of adjacent rows) given the linear planting process, and thus lead thereafter to symptomatic rows of strawberry plants. Secondary infections due to overhead irrigation (used to promote plant establishment and growth) or rainfall events have too steep dispersal gradients to allow bacteria to easily disperse from bed to bed in strawberry fields. Conversely, crop operations along the rows or beds (i.e., mainly the practice of flower removal which is characterized by the systematic handling of every plant along the rows) make it possible to disperse the pathogen in the same direction, regularly, throughout the growing season. It seems, therefore, essential to take into account recurrent crop husbandry operations (such as plant trimming) to design improved control strategies. Finally, even though it was relatively rare (two cases over the 97 data sets), we saw that border effects may led to very high degrees of aggregation. The field assessments in question were characterized by the presence of an adjacent upwind strawberry field with a high ALS intensity. We can assume that the water-dispersed pathogen was carried over longer distances because of the drift experienced by overhead irrigation in the windy area where these fields were located. That would explain why up to four beds adjacent to the upwind highly infected field exhibited higher disease intensity in comparison with the other beds (Fig. 1C). Under such environmental conditions, using antidrift nozzles and planting highly susceptible cultivars downwind could minimize such border effects.

The characterization and quantification of ALS patterns made it possible to identify major factors favoring disease progression in California nursery field conditions. In particular, crop husbandry operations along agronomic rows appear to play a significant role in disease spread which is all the more important given the recurrence of such operations over the growing season. The new insights about ALS epidemiology provide strawberry nurseries with the foundational information to make informed decisions on revamping their ALS management practices. In the future, it would be valuable to get quantitative information about the long-term survival of $X$. fragariae under field conditions, and the role of asymptomatic infected plants and how they contribute to initial inoculum. Indeed, a more comprehensive understanding of these two epidemiological features should offer further insight on how to improve disease control.

\section{ACKNOWLEDGMENTS}

We thank the California nurseries involved in this project, in particular for their close collaboration over the years. We also acknowledge the California Strawberry Nursery Growers Association. We thank the two anonymous referees for their constructive comments on a previous version of this manuscript. This work received financial support from USDA-FAS Technical Assistance for Specialty Crops (TASC Grant 2012-51).

\section{LITERATURE CITED}

Bestfleisch, M., Richter, K., Wensing, A., Wünsche, J. N., Hanke, M. V., and Höfer, M. 2015. Resistance and systemic dispersal of Xanthomonas fragariae in strawberry germplasm (Fragaria L.). Plant Pathol. 64:71-80.

Garrett, K. A., and Mundt, C. C. 1999. Epidemiology in mixed host populations. Phytopathology 89:984-990.

Haldane, J. B. S. 1956. The estimation and significance of the logarithm of a ratio of frequencies. Ann. Hum. Genet. 20:309-311.

Horne, J. K., and Schneider, D. C. 1995. Spatial variance in ecology. Oikos 74: 321-329.

Hughes, G., and Gottwald, T. R. 1999. Survey methods for assessment of Citrus tristeza virus incidence when Toxoptera citricida is the predominant vector. Phytopathology 89:487-494.

Hughes, G., Gottwald, T. R., and Levy, L. 2002. The use of hierarchical sampling in the surveillance program for plum pox virus incidence in the United States. Plant Dis. 86:259-263.

Hughes, G., and Madden, L. V. 1992. Aggregation and incidence of disease. Plant Pathol. 41:657-660.

Hughes, G., and Madden, L. V. 1993. Using the beta-binomial distribution to describe aggegated patterns of disease incidence. Phytopathology 83: 759-763.

Hughes, G., McRoberts, N., Madden, L. V., and Gottwald, T. R. 1997. Relationships between disease incidence at two levels in a spatial hierarchy. Phytopathology 87:542-550.

Kennedy, B. W., and King, T. H. 1962. Angular leaf spot of strawberry caused by Xanthomonas fragariae sp. nov. Phytopathology 52:873-875.

Li, B., Madden, L. V., and Xu, X. 2012. Spatial analysis by distance indices: An alternative local clustering index for studying spatial patterns. Methods Ecol. Evol. 3:368-377.

Lin, L. I.-K. 1989. A concordance correlation coefficient to evaluate reproducibility. Biometrics 45:255-268

Lin, L. I.-K. 2000. A note on the concordance correlation coefficient. Biometrics 56:324-325.

Maas, J. L. (ed.) 1998. Compendium of Strawberry Diseases. 2nd ed. American Phytopathological Society, St. Paul, MN.

Maas, J. L., Pooler, M. R., and Galletta, G. J. 1995. Bacterial angular leafspot disease strawberry: Present status and prospects for control. Adv. Strawberry Res. 14:18-24.

Madden, L. V., and Hughes, G. 1995. Plant disease incidence: Distributions, heterogeneity, and temporal analysis. Annu. Rev. Phytopathol. 33:529-564.

Madden, L. V., and Hughes, G. 1999. An effective sample size for predicting plant disease incidence in a spatial hierarchy. Phytopathology 89:770-781.

Madden, L. V., Hughes, G., and Ellis, M. A. 1995a. Spatial heterogeneity of the incidence of grape downy mildew. Phytopathology 85:269-275.

Madden, L. V., Hughes, G., and van den Bosch, F. 2007. The Study of Plant Disease Epidemics. American Phytopathological Society, St Paul, MN.

Madden, L. V., Nault, L. R., Murral, D. J., and Apelt, M. R. 1995b. Spatial pattern analysis of the incidence of aster yellows disease in lettuce. Res. Popul. Ecol. (Kyoto) 37:279-289.

Oehlert, G. W. 1992. A note on the delta method. Am. Stat. 46:27-29.

Perry, J. N. 1995. Spatial analysis by distance indices. J. Anim. Ecol. 64:303-314.

Perry, J. N., Winder, L., Holland, J. M., and Alston, R. D. 1999. Red-blue plots for detecting clusters in count data. Ecol. Lett. 2:106-113.

Pooler, M. R., Ritchie, D. F., and Hartung, J. S. 1996. Genetic relationships among strains of Xanthomonas fragariae based on random amplified polymorphic DNA PCR, repetitive extragenic palindromic PCR, and enterobacterial repetitive intergenic consensus PCR data and generation of multiplexed PCR primers useful for the identification of this phytopathogen. Appl. Environ. Microbiol. 62:3121-3127.

Reynolds, K. M., and Madden, L. V. 1988. Analysis of epidemics using spatiotemporal autocorrelation. Phytopathology 78:240-246.

Reynolds, K. M., Madden, L. V., and Ellis, M. A. 1988. Spatio-temporal analysis of epidemic development of leather rot of strawberry. Phytopathology 78:246-252.

Roberts, P. D., Berger, R. D., Jones, J. B., Chandler, C. K., and Stall, R. E. 1997. Disease progress, yield loss, and control of Xanthomonas fragariae on strawberry plants. Plant Dis. 81:917-921.

Roberts, P. D., Hodge, N. C., Bouzar, H., Jones, J. B., Stall, R. E., Berger, R. D. 1998. Relatedness of strains of Xanthomonas fragariae by restriction fragment length polymorphism, DNA-DNA reassociation, and fatty acid analyses. Appl. Environ. Microbiol. 64:3961-3965.

Sparks, A. H., Esker, P. D., Antony, G., Campbell, L., Frank, E. E., Huebel, L., Rouse, M. N., Van Allen, B., and Garrett, K. A. 2008. Ecology and epidemiology in R: Spatial analysis. The Plant Health Instructor. doi:10.1094/ PHI-A-2008-0129-03

Stevenson, M., Nunes, T., Heuer, C., Marshall, J., Sanchez, J., Thornton, R. 2016. epiR: Tools for the analysis of epidemiological data. Published online. https://cran.r-project.org/web/packages/epiR/ 
Taylor, L. R. 1961. Aggregation, variance and the mean. Nature 189:732-735.

Turechek, W. W., Ellis, M. A., and Madden, L. V. 2001. Sequential sampling for incidence of Phomopsis leaf blight of strawberry. Phytopathology 91:336-347.

Turechek, W. W., and Madden, L. V. 1999a. Spatial pattern analysis and sequential sampling for the incidence of leaf spot on strawberry in Ohio. Plant Dis. 83:992-1000.

Turechek, W. W., and Madden, L. V. 1999b. Spatial pattern analysis of strawberry leaf blight in perennial production systems. Phytopathology 89:421-433.

Turechek, W. W., and Madden, L. V. 2003. A generalized linear modeling approach for characterizing disease incidence in a spatial hierarchy. Phytopathology 93:458-466.

Turechek, W. W., Madden, L. V., Gent, D. H., and Xu, X.-M. 2011. Comments regarding the binary power law for heterogeneity of disease incidence. Phytopathology 101:1396-1407.

Turechek, W. W., and Mahaffee, W. F. 2004. Spatial pattern analysis of hop powdery mildew in the Pacific Northwest: Implications for sampling. Phytopathology 94:1116-1128.
Turechek, W. W., and McRoberts, N. 2013. Considerations of scale in the analysis of spatial pattern of plant disease epidemics. Annu. Rev. Phytopathol. 51:453-472.

Turechek, W. W., and Peres, N. A. 2009. Heat treatment effects on strawberry plant survival and angular leaf spot, caused by Xanthomonas fragariae, in nursery production. Plant Dis. 93:299-308.

Turechek, W. W., Wang, S., Tiwari, G., and Peres, N. A. 2013. Investigating alternative strategies for managing bacterial angular leaf spot in strawberry nursery production. Int. J. Fruit Sci. 13:234-245.

van der Gaag, D. J., Bergsma-Vlami, M., van Vaerenbergh, J., Vandroemme, J., and Maes, M. 2013. Pest risk analysis for Xanthomonas fragariae. Netherlands Food and Consumer Product Safety AuthorityMinistry of Economic affairs, Agriculture and Innovations, the Netherlands.

Wang, H., and Turechek, W. W. 2016. A loop-mediated isothermal amplification assay and sample preparation procedure for sensitive detection of Xanthomonas fragariae in strawberry. PLoS One 11:e0147122. 